\title{
Discover Wellness: A Worksite Wellness Program for Higher Education
}

\author{
Elizabeth Keida \\ State University of New York at Oswego \\ Jessica Harris \\ State University of New York at Oswego \\ Barry A. Friedman \\ State University of New York at Oswego
}

\begin{abstract}
Sixty percent of adults in the United States suffer from chronic disease. Worksite wellness programs that target at-risk populations have positive health benefits. Discover Wellness: Find a Healthier You (DW) is a worksite wellness program intended to improve the higher education employee health by providing an opportunity for participants to learn and practice healthy behaviors. The purpose of this study was to evaluate DW program impact on employee behavior change to reduce chronic disease risk. This study employed a pretest-posttest quasi-experimental design that assessed self-efficacy and health behaviors of employees of a state university in the northeast United States. Participants experienced significant improvement in stress $\left(t_{23}=-31.602, p<0.001\right)$, nutrition $\left(t_{21}=-36.313, p<0.001\right)$, physical activity $\left(t_{22}\right.$ $=-34.380, p<0.001)$, and sleep $\left(t_{23}=-18.450, p<0.001\right)$. Additionally, anecdotes from participants revealed themes of comradery and reflection on health behaviors.
\end{abstract}

Keywords: worksite wellness, health promotion, higher education

\section{INTRODUCTION}

According to the Centers for Disease Control (CDC), six in ten Americans are living with at least one chronic disease such as heart disease, stroke, cancer, or diabetes (CDC, 2021). These chronic health conditions adversely impact individual well-being and places a massive burden on the U.S. economy with annual healthcare costs totaling \$3.5 trillion (CDC, 2021). Chronic diseases significantly impact individuals' work productivity, absenteeism, and employer's healthcare costs (Nazarov et al., 2019). With health care costs on the rise, worksite health promotion programs (WHPP) can slow this upward trend to reduce employees' risks of developing a chronic disease. Disruptive events such as pandemics exacerbate the adverse impacts to employees, unemployment, workplace costs, lost productivity, as well as adverse macroeconomic impacts to the global economies (Cheong et al, 2020; Pak et al, 2020). These adverse impacts make workplace health promotion programs even more essential.

Chronic diseases, such as obesity and heart disease can be prevented and severity can be improved through positive health behaviors (Haplin et al., 2010; Willett et al., 2006). Worksite wellness programs are ideal for targeting populations at risk for such diseases and have been shown to have positive health benefits 
(Aldana et al., 2005; CDC, 2019). WHPPs are employer-based training programs aimed to improve the health of employees (CDC, 2019). The main focus of these programs is to reduce the risk of chronic disease and, in turn, improve employee productivity. According to a review conducted by Heaney and Goetzel (1997), programs that were long term and conducted risk reduction counseling were critical in an effective worksite wellness program. WHPP, in general, have been shown to decrease health risk factors and generate savings within the worksite by reducing healthcare and absenteeism costs and improving worker productivity (CDC, 2019).

Notoriously, health promotion programs on college campuses have been geared towards the students, and as of 2016, $57 \%$ of colleges and universities have reported offering WHPPs for employees (Bichsel \& McChesney, 2017). The college setting offers an ideal venue for successful behavior and lifestyle changes designed to improve health, prevent chronic disease onset, and lessen severity. Higher education worksites have an infrastructure conducive for wellness program success including an established communication network and social networks, and access to fitness centers and other health promotion facilities (Hill-Mey et al., 2015; Linnan et al., 2017). College campuses are the optimal locations to target a large number of employees to improve health and decrease rates of chronic disease.

The purpose of this study is to ascertain the effectiveness of a wellness intervention intended to improve the health of employees in higher education. Specifically, the researchers studied the Discover Wellness: Find a Healthier You worksite wellness program's impact on employee behavior change to combat risks of chronic diseases. The researchers first report the research methodology used presently (research design, program implementation, sample, data analysis) followed by the results. The researchers conclude with study implications, limitations, and future research.

\section{RESEARCH DESIGN}

Discover Wellness: Find a Healthier You $(D W)$ was a virtual worksite health promotion program targeting employees in higher education. With many universities moving towards a wellness culture it is imperative to offer programming to critical members of our campus community; employees. The $D W$ program was developed as a result of a needs assessment survey that was administered during the fall of 2018 to investigate the health and wellness of employees at a state university in the northeast United States. A needs assessment is a critical component of complete worksite health promotion programs to establish a rationale (CDC, 2016; Hill-Mey et al., 2015). The program aimed to improve employees' overall health, reduce chronic disease risk, promote a strong wellness culture, and improve employee productivity. This research had been approved by the university's Institutional Review Board.

FIGURE 1

SEVEN WEEK IMPLEMENTATION PROCESS

\begin{tabular}{|c|c|c|c|c|}
\hline Pre-Survey & \multicolumn{3}{|c|}{ Intervention } & Post-Survey \\
\hline Data collection & Monday & Tuesday-Thursday & Friday & Data collection \\
\cline { 2 - 4 } & $\begin{array}{c}10 \text { minutes: Mini- } \\
\text { Lecture (teach) } \\
\begin{array}{c}45 \text { minutes: Activity } \\
(d o)\end{array}\end{array}$ & $\begin{array}{c}\text { Implement behavior } \\
\text { change techniques } \\
(d o)\end{array}$ & $\begin{array}{c}60 \text { minutes: } \\
\text { Group Wellness } \\
\text { Coaching } \\
(\text { reflect })\end{array}$ & \\
\hline
\end{tabular}

\section{Program Implementation}

$D W$ was implemented at a northeast state university in the United States. The program was seven weeks in duration, and participants met virtually twice a week to work on improving specific health behaviors (stress management, nutrition, physical activity, and sleep) through a teach-do-reflect model (See Figure 
1). During the first meeting of the week, participants engaged in a mini-lecture (teach) followed by a handson activity $(d o)$. At the end of the week, participants met as a group with a certified wellness coach to discuss their behavior change progress (reflect).

\section{Sample}

Participants were volunteers from a northeast state university in the United States $(\mathrm{N}=13)$. All participants were female and their ages ranged in all predetermined categories (26-65 years). Twelve of the participants were professional staff and one participant was a faculty member.

\section{Survey}

The survey consisted of nine sections which included demographics, stress, nutrition, physical activity, sleep, behavior change, experience, and wellness coaching. To ensure anonymity and obtain unbiased results the demographic section was limited to a unique identifier, age, and gender.

Self-efficacy and health behaviors were measured before and after implementation of the program using Likert scales for five of the sections. There were five questions related to stress, eight questions related to nutrition, four questions about sleep, and seven questions related to physical activity, specifically resistance training and functional movement. A typical question was "I know about strategies/tools that I can use to help improve my sleep." Participants had the choice to respond with "never, almost never, sometimes, fairly often, or very often." This part of the survey enabled the researchers to investigate each area of health and measure the participants' change. The stress section measured the ability of participants to identify and manage stress. The physical activity section includes the confidence level and ability to engage in physical activity. The sleep section measured whether participants obtained the recommended amount of sleep each night (7-8 hours) and knowledge and awareness of sleep strategies. The nutrition section measured the participants' knowledge of the adhering to the FDA guidelines for sodium, cholesterol and sugar intake.

Sections 6-8 were only part of the post-survey and were implemented following the completion of the program. These questions were open ended questions that allowed participants to reflect on their own health behaviors and experience in the wellness program and group coaching sessions.

\section{Data Analysis}

IBM SPSS Statistics (v. 27) was used to analyze the data. Four paired sample t tests were conducted to determine if there were significant differences between the before and after assessment with respect to the employees' average rating of health skill and knowledge after they completed the seven-week program.

\section{RESULTS}

One hundred percent of participants successfully completed the program $(N=14)$. The attendance rate for the program was $79 \%$. Of the 98 possible sessions, where there were seven sessions for 14 participants, there were only 21 absences). This does not include the 98 coaching sessions, where the attendance rate was $66 \%$ (34 absences). Thirteen participants completed the pre-survey and 11 completed the post survey allowing us to run the analysis with 24 sets of data. All participants who participated in the post-survey $(N$ $=11$ ) stated that they continued to implement the health behaviors into their daily lives.

Due to the mean of the two scores, and the direction of the $t$ value, we can conclude that all four of the variables showed statistically significant improvements in employees' health behaviors related to stress nutrition, sleep and physical activity following participation in $D W(p<0.001)$. Specifically, there was a significant mean difference between pre and post scores for stress $\left(t_{23}=31.602, p<0.001\right)$, physical activity $\left(t_{22}=34.380, p<0.001\right)$, sleep $\left(t_{23}=18.450, p<0.001\right)$ and nutrition $\left(t_{21}=36.313, p<0.001\right)$. Additionally, anecdotal data provided evidence that participants benefited from the comradery and reflection of their health behaviors. 
TABLE 1

PRE AND POST MEAN DIFFERENCE AND t-TEST RESULTS

\begin{tabular}{lcccccccc}
\hline & \multicolumn{3}{c}{ Pre-Test } & \multicolumn{7}{c}{ Post-Test } \\
& $\mathrm{M}$ & $(\mathrm{N})$ & $\mathrm{SD}$ & $\mathrm{M}$ & $\mathrm{(N)}$ & $\mathrm{SD}$ & $\mathrm{df}$ & $\mathrm{t}$ \\
\hline Stress & 14.384 & $(13)$ & 2.328 & 16.181 & $(11)$ & 1.887 & 23 & $-31.602^{*}$ \\
Physical Activity & 24.384 & $(13)$ & 3.123 & 27.500 & $(10)$ & 3.503 & 22 & $-34.380^{*}$ \\
Sleep & 12.307 & $(13)$ & 3.591 & 13.636 & $(11)$ & 2.149 & 23 & $-18.405^{*}$ \\
Nutrition & 26.750 & $(12)$ & 5.801 & 34.600 & $(10)$ & 4.221 & 21 & $-22.121^{*}$ \\
\hline${ }^{*} p<.001$ & & & & & & & &
\end{tabular}

A thematic analysis was done in order to ascertain participants' perspectives regarding their continued use of strategies, tools, and experiences regarding the wellness coaching sessions. The researchers also intended to gain further insight into how participants viewed program implementation. Based on what participants were taught in the program, several common themes were stated about their continued use of these tools and strategies, such as (1) stress reduction strategies, (2) physical activity, (3) nutritional improvements, (4) sleep schedules, which were all consistent with the targeted health behaviors during $D W$. Participants also offered feedback regarding additional tips and tools needed to further facilitate their use of the skills learned in $D W$, such as having (1) accountability, (2) use of technology for reminders, (3) continued knowledge on how to implement the tools, and (4) access to supplemental materials as resources. Participants further identified that their experiences with the coaching sessions provided them with (1) positive affirmations, (2) inspired inquiry, and (3) helped reframe their experiences. Participants did further identify that there were (4) time constraints that prohibited many from attending these coaching sessions.

The qualitative data revealed that participants were successful in their behavior changes. However, all participants did state that they had areas that they wanted to continue to improve on. They explained that they knew what they needed to do, however it was either a lack of motivation or injury that prevented them from participating. This indicated a strong self-efficacy and knowledge, just limited motivation.

\section{CONCLUSION}

The $D W$ program was implemented with state university employees as an intervention for health behavior change wellness. Pre-test and post-test measures revealed that participants experienced significant improvement in stress reduction, sleep, physical activity and nutrition at the conclusion of the program. Further analysis of participants' comments following the program found that they were engaged and responded favorably to the intervention.

Providing an opportunity for participants to learn and practice healthy behaviors may be the key to behavior change. Increasing these opportunities in populations, such as university employees, may reduce rates of chronic diseases. A full-time employee spends $50 \%$ of their day at work, having a wellness culture in higher education increases employee well-being and the quality of work completed (Rose et al., 2017). For comprehensive worksite wellness programs to succeed, administrators must adopt strategies to get the majority of its faculty and staff to participate in to get a desired outcome (Rose et al., 2017) that (1) improves employee health behaviors, (2) reduces elevated health risks, (3) reduces health costs, (4) improves productivity, (5) decreases absenteeism, (6) improves employee recruitment and retention, and (7) overall builds and helps sustain high employee morale on college campuses (Aldana, 2020)

\section{Study Limitations}

Like all research, this study had limitations concerning sample size, generalizability and design. The small sample limited statistical power and generalizability to the population of university employees. Regarding generalizability, the study employed a convenience sample of all female volunteers, thus limiting generalizing the findings to other populations not included in the present study (e.g., males, minorities,and 
non-staff employees). Generalizability to other organizations and workplaces beyond academia is also limited. Additionally, the longer-term impact of wellness and healthy behaviors was not ascertained. Finally, a control group was not deployed in the present quasi experimental design.

\section{Implications and Future Research}

The study limitations described above have implications for future research. Larger and more diverse samples drawn from several workplace settings should be conducted (e.g., profit, non-profit, manufacturing. consulting, service, government, private sector). Longitudinal research should track behavior change and health over time. An experimental design complete with randomization to condition and control should be deployed. The COVID-19 pandemic also poses a profound challenge in regard to sedentary lifestyle, making it even more important for college-level administrators to engage their employees in remote opportunities that encourage wellness. The future of worksite wellness must address new issues such as mental health in the same way it addresses nutrition, fitness, and chronic conditions to meet the needs of employees during future unprecedented times.

\section{ACKNOWLEDGEMENTS}

We would like to acknowledge the Discover Wellness instructors who taught the sessions during the program: Daniel Hotaling, Whitney Kmetz, Richard McCabe, Gabriella Schaff, Anne Seichepin, and Shannon Stone. We would also like to acknowledge the Certified Wellness Coach who led the group coaching sessions each week: Dr. Amy Bidwell.

\section{REFERENCES}

Aldana, S. (2020). The 7 best reasons to have a wellness program: Benefits of wellness. Well Steps. Retrieved from https://www.wellsteps.com/blog/2020/01/02/reasons-to-have-a-wellnessprogram-benefits-of-wellness/

Aldana, S.G., Greenlaw, R.L., Diehl, H.A., Salberg, A., Merrill, R,M., \& Ohmine, S. (2005). The effects of a worksite chronic disease prevention program. Journal of Occupational and Environmental Medicine, 47(6), 558-564. Retrieved from https://journals.lww.com/joem/Abstract/2005/06000/The_Effects_of_a_Worksite_Chronic_Disea se.6.aspx

Bichsel, J., \& McChesney, J. (2017). Employee healthcare benefits in higher education: Key findings and summary tables for the 2016-17 academic year. Research report. Knoxville, TN: CUPA-HR. Retrieved from http://www.cupahr.org/surveys/benefits.aspx

Center for Disease Control and Prevention. (2019, May 29). CDC: Half of workplaces offer health/wellness programs. US Department of Health and Human Services. Retrieved from https://www.cdc.gov/media/releases/2019/p0422-workplaces-offer-wellness.html

Center for Disease Control and Prevention. (2016). Workplace health model. US Department of Health and Human Services. Retrieved from https://www.cdc.gov/workplacehealthpromotion/model/index.html

Center for Disease Control and Prevention. (2019). Workplace health promotion. US Department of Health and Human Services. Retrieved from https://www.cdc.gov/workplacehealthpromotion/index.htm

Center for Disease Control and Prevention. (2021, January 12). About chronic diseases. US Department of Health and Human Services. Retrieved from https://www.cdc.gov/chronicdisease/about/index.htm

Cheong, K.H., Wen, T., \& Lai, J.W. (2020). Relieving cost of epidemic by parrondo's paradox: A COVID-19 case study. Advanced Science, 7(24), 2002324-n/a. https://doi.org/10.1002/advs.202002324 
Halpin, H.A., Morales-Suárez-Varela, M.M., \& Martin-Moreno, J.M. (2010). Chronic disease prevention and the new public health. Public Health Reviews, 32(1), 120-154. Retrieved from https://publichealthreviews.biomedcentral.com/track/pdf/10.1007/BF03391595.pdf

Heaney, C.A., \& Goetzel, R.Z. (1997). A review of health-related outcomes of multi-component worksite health promotion programs. Am J Health Promot., 11(4), 290-307. https://doiorg.ezproxy.oswego.edu/10.4278/0890-1171-11.4.290

Hill-Mey, P., Hyatt-Neville, B., Kumpfer, K., Merrill, R., Reel, J., \& Richardson, G. (2015). Worksite health promotion programs in college settings. Journal of Education and Health Promotion, 4(1), 12. Retrieved from https://www.ncbi.nlm.nih.gov/pmc/articles/PMC4389364/

IBM Corp. (2020). IBM SPSS Statistics for Windows, Version 27.0. Armonk, NY: IBM Corp.

Linnan, L.A., Arandia, G., Nasser, C., Li, J., Pomerantz, M., \& Diehl, S.J. (2017). Assessing opportunities to enhance comprehensive health promotion and wellness programming in a state community college system. North Carolina Medical Journal, 78(5), 296-303. https://doi.org/10.18043/ncm.78.5.296

Nazarov, S., Manuwald, U., Leonardi, M., Silvaggi, F., Foucaud, J., Lamore, K., . . Rothe, U. (2019). Chronic diseases and employment: Which interventions support the maintenance of work and return to work among workers with chronic illnesses? A systematic review. International Journal of Environmental Research and Public Health, 16(10), 1864. Retrieved from https://www.ncbi.nlm.nih.gov/pmc/articles/PMC6572561/

Pak, A., Adegboye, O.A., Adekunle, A.I., Rahman, K.M., McBryde, E.S., \& Eisen, D.P. (2020). Economic consequences of the COVID-19 outbreak: The need for epidemic preparedness. Frontiers in Public Health, 8, 241. https://doi.org/10.3389/fpubh.2020.00241

Proper, K.I., \& Van Oostrom, S.H. (2019). The effectiveness of workplace health promotion interventions on physical and mental health outcomes: A systematic review of reviews. Sc and J Work Environ Health, 45(6), 546-559. Retrieved from https://www.sjweh.fi/show_abstract.php?abstract_id=3833\&fullText=1

Rose, A., Lakhese, K., Zettervall, A., Robertson, W., \& Walden, B. (2017). Developing university wellness programs. Retrieved from https:/hr.unc.edu/wpcontent/uploads/sites/222/2017/12/Developing-University-Wellness-Programs-ULEAD-2017.pdf

Willett, W.C., Koplan, J.P., Nugent, R., Dusenbury, C., Puska, P., \& Gaziano, T.A. (2006). Prevention of chronic disease by means of diet and lifestyle changes. In Disease Control Priorities in Developing Countries (2nd edition). Retrieved from https://www.ncbi.nlm.nih.gov/books/NBK11795/ 